\title{
La communication par SMS: quoi de neuf?
}

\section{SMS communication: what's new?}

\author{
Mercedes Banegas Saorín \\ Université Polytechnique Hauts-de-France \\ Unité de Recherche DeSCRIPTO \\ mercedes.banegassaorin@uphf.fr
}

\begin{abstract}
This study aims to show, from a sociolinguistic perspective, the trends of the language of instant messaging developped by young generationts, The goal is to determine how innovative this language practice is. To do this, we observe the formal typologies of the written SMS established between 2001 and 2017 by researchers in linguistics, while verifying that this mode of communication is inscribed within certain natural behaviors of human language.
\end{abstract}

\section{Key words}

SMS communication, graphic variation, youngs'sociolect.

\section{Resumen}

Este estudio pretende mostrar, desde una perspectiva sociolingüística, las tendencias del lenguaje de la mensajería instantánea. Desarrollado por las jóvenes generaciones, el objetivo es determinar hasta qué punto esta práctica lingüística es innovadora. Para ello, observamos las tipologías formales de la escritura SMS establecidas entre 2001 y 2017 por los investigadores en lingüística al tiempo que comprobamos que este modo de comunicación se inscribe dentro de ciertos comportamientos naturales del lenguaje humano.

\section{Palabras clave}

comunicación por SMS, variación gráfica, sociolecto juvenil. 


\section{Introduction et corpus de base}

La communication de la messagerie instantanée est largement nommée "langage des textos", "l'eSMS" (l'écriture SMS) et “communication médiée par téléphone portable". Née lors de la révolution numérique, elle a déjà plus de vingt ans d'existence. Cette pratique linguistique, qui s'écarte de la langue écrite conventionnelle, n'est pas réservée aux messages envoyés par téléphone car aujourd'hui, les utilisateurs emploient le langage SMS dans toutes les discussions en ligne, qu'elles soient synchrones ou asynchrones, et sur toutes les applications de messagerie instantanée, que ce soit WhatsApp, SnapChat ou les réseaux sociaux ${ }^{1}$. Ce langage a pu élargir ses ressources sémiotiques avec l'insertion d'émoticônes et de photos et, pourtant, il a quelque peu évolué depuis les années 2000, lorsque les claviers avaient 12 touches alphanumériques ${ }^{2}$ et les SMS étaient limités à 160 caractères pour les alphabets latins, ce qui poussait les scripteurs au gain d'espace pour réduire le coût de la communication; ceci se traduisait par l'emploi de chiffres dont la valeur phonétique équivaut à une syllabe ("7 swaré" pour "cette soirée") ou encore par la suppression de lettres muettes ("drol" pour “drôle”). Depuis 2007, les téléphones portables ont évolué vers des smartphones dotés d'un écran tactile et des logiciels d'écriture intuitive et certaines de ces pratiques ont décliné. Mais le nombre de SMS échangés quotidiennement est grandissant. Les indices révélateurs de l'ampleur du phénomène sont les suivants:

- Le langage SMS a inévitablement fait irruption dans le monde académique : depuis ses débuts il a commencé à être traité dans des congrès et des séminaires.

- Des plateformes en ligne pour apprendre le français général et de spécialité tiennent compte de la modalité SMS dans les fiches pédagogiques des professeurs. $^{3}$

- Certaines entreprises de plusieurs pays (France ${ }^{4}$, Royaume Uni ${ }^{5}$, Argentine ${ }^{6}$ ) proposent de postuler à une offre d'emploi via des SMS.

1 Il serait opportun d'inclure la communication SMS, avec Marcoccia (2016), dans un domaine plus large: celui des écrits numériques formés aussi de courriers électroniques, forums, blogs qui, tout en ayant des points en commun, diffèrent d'un texto selon divers paramètres tels que le type et nombre d'écarts par rapport à l'écrit standard.

2 Les messages SMS étaient encodés avec la "saisie T9", appelée aussi "saisie ou frappe intuitive ou prédictive". Cette technologie consistait à assigner plusieurs lettres à chaque touche. Pour accéder à chacune d'entre elles il fallait appuyer un nombre de fois déterminé par l'ordre alphabétique et le nombre de lettres associées à chaque touche. On simplifiait, ainsi, la saisie de texte sur les claviers téléphoniques.

3 La plateforme Bonjour de France, créée en 1998, propose une leçon de niveau B2 ayant pour titre "Le langage SMS ? KskC?”. Cf. http://www.bonjourdefrance.com/exercices/contenu/19/vocabulaire/547.html

$4 \quad C f$. https://www.clever.fr/autres-liens/recrutement-sms/

$5 \quad C f$. https://www.journaldunet.com/management/ressources-humaines/1069468-postuler-a-une-offre-d-emploipar-sms/

6 Cf. Journal argentin Clarín du 15/01/2008. Accessible sur https://www.clarin.com/ediciones-anteriores/cvsms_0_Sy7NA80RaKg.html. 
- Malgré la nature mouvante de ce style de langage, des glossaires SMS circulent sur internet mais aussi des publications imprimées?

- Des plateformes font du marketing mobile à destination d'un consommateur, de façon ciblée, dans les secteurs de l'immobilier, de la mode, de la finance, de la restauration ${ }^{8}$.

- Les SMS investissent aussi les journaux et sont source de création littéraire: non seulement ils font l'objet de nombreux articles de presse, mais, de plus, un projet de recueil de SMS d'amour, lancé sur Instagram ${ }^{9}$, a abouti à un roman, Amours solitaires (2018), où l'auteure, M. Ortin, puise la trame dans les 25000 messages reçus depuis la création du compte Instagram.

Ce phénomène a soit inquiété soit réjoui le grand public et surtout parents, enseignants, linguistes, sociologues, psychologues et autres spécialistes de la communication. Les plus réticents pensent que cette pratique nuit à l'apprentissage de la grammaire et de l'orthographe des langues. Les plus positifs considèrent que l'usage de ce code disorthographique ou néographique n'est qu'une nouvelle forme d'expression enrichissante pour les échanges entre individus. Ainsi, un groupe de chercheurs du Centre de recherche sur la cognition et l'apprentissage (CeRCA, université de Poitiers) ${ }^{10}$, ont montré en 2014 que le langage SMS n'exerce pas une influence négative sur l'orthographe des collégiens. Cette étude, menée sur près de 5000 SMS rédigés par 19 collégiens de $6^{\mathrm{e}}$ et de $5^{\mathrm{e}}$, âgés de 11 à 12 ans, révèle que ce serait l'orthographe traditionnelle qui influe sur la pratique des SMS, lesquels, d'ailleurs, incitent les jeunes à pratiquer l'écrit.

Des thèses de doctorat continuent de nourrir la recherche actuelle dans les domaines de la linguistique et de la psycholinguistique: celle de C. Combes (2014) s'intéresse au coût cognitif de l'écriture SMS chez les adolescents; F. André (2017) étudie les différents paramètres qui déterminent les pratiques scripturales des SMS (âge, situation...); É. Morel (2017) s'intéresse aux spécificités des pratiques plurilingues dans la communication par texto ; la thèse de E. Kogkitsidou (2018) observe les écarts grammaticaux des SMS par rapport à la langue standard et propose un modèle de normalisation.

Des classifications très exhaustives sur les caractéristiques scripturales des textos ont été faites par plusieurs groupes de chercheurs, notamment en sciences du langage: Anis (2001, 2004), Fairon et al. (2006), Liénard (2008), Panckhurst (2009), Cougnon (2010), Fairon \& Klein (2010), Cougnon \& François (2011), Panckhurst \& Moïse (2012), Morel \& Pe-

7 À titre d'exemple: Éric Poussevy (2010, réed. 2012), Parlez-vous le SMS?: dictionnaire bilingue français-SMS, Paris, L'Express, et D\&V Translation Agency: https://www.dvtranslation.com/blog/apprendre-le-langage-smsen-trois-etapes/

8 Cf. http://marketing-mobile.com/

$9 C f$. Duretz dans sitographie.

10 CeRCA - CNRS, UMR 7295. Auteurs : Bernicot, Goumi, Bert-Erboul et Volckaert-Legrier (2014), cf. bibliographie. 
karek Doehler (2013), Panckhurst et al., (2014). D’ailleurs, le projet européen SMS4science, mené par l'Université Stendhal, à Grenoble, et le Conseil Général des Hautes-Alpes, a fait suite au premier projet-pilote belge "Faites don de vos SMS à la science", fondé en 2004 par le CENTAL et le CELEXROM à l'Université Catholique de Louvain. Il recouvre six zones géographiques: la Belgique francophone (75 000 SMS dont 30000 SMS exploités dans la base de données sms4science, Fairon et al., 2006), La Réunion (12 000 SMS), la Suisse (24 000 SMS), le Québec (5 000 SMS), les Hautes-Alpes et l'Isère (22 000 SMS) et le Languedoc-Roussillon (93 $114 \mathrm{SMS})^{11}$.

Ces collectes ont permis de réunir des données authentiques dans la mesure où ce sont de véritables messages échangés entre usagers du SMS et non élaborés artificiellement à des fins d'enquête. C'est pourquoi nous nous appuyons sur les classements formels qu'ont fournis ces chercheurs à partir desdits corpus pour mener à bien notre réflexion.

Dans les pages qui suivent nous essayons de montrer les tendances du langage de la messagerie instantanée développé par les jeunes générations. Resté en marge de la norme, a continué d'être développé par les adolescents mais aussi par certains jeunes adultes en situation informelle. Le but que nous nous sommes fixé est de déterminer dans quelle mesure cette pratique langagière est innovante. Pour ce faire, nous observons les typologies formelles de l'écriture SMS élaborées entre 2001 et 2017 par des chercheurs en linguistique tout en ancrant ce mode de communication dans certains comportements naturels du langage humain.

Tous les classements existants rendent compte des variations de l'écriture SMS par rapport à l'écrit normé, décrites comme disorthographiques, néographiques ou étant des "déviances" de la langue écrite.

Lorsque le changement des langues est devenu l'objet scientifique de la linguistique historique, au XIX ${ }^{\mathrm{e}}$ siècle, les linguistes ont constaté que cette transformation obéit aussi bien à l'intentionnalité des hommes qu'à une nécessité interne (Ducrot \& Todorov (1972: 20):

Le changement des langues n'est pas dû seulement à la volonté consciente des hommes (effort d'un groupe pour se faire comprendre d'étrangers, décision des grammairiens qui "épurent" le langage, création de mots nouveaux pour désigner des idées nouvelles) mais aussi à une nécessité interne.

Voyons ce que révèlent les changements propres à l'eSMS par rapport au français standard qui, d'ailleurs, sont instillés dans des proportions diverses dans la langue circulante. Même si de multiples phénomènes apparaissent simultanément dans un même message, des typologies détaillées ont été établies dans les travaux précédemment cités. Or, il est possible de regrouper ces marques en suivant les tendances générales qui ressortent afin de dégager les caractéristiques linguistiques les plus saillantes de cette forme de communication.

11 Pour plus d'informations, voir http://www.sms4science.org et http://www.uclouvain.be/cental. 


\section{Phonétisation des graphies}

Parmi les variations qui ont été relevées par les auteurs cités dans l'introduction, il $\mathrm{y}$ a tout un ensemble de phénomènes néographiques qui affichent une claire tendance à la phonétisation des caractères. Une description globale et synthétique pourrait être la suivante:

- remplacement d'un son par une lettre unique, avec la valeur sonore qu'on lui confère quand on la dénomme: Je t'm (je t'aime); CT (c'était), h.e.t ou $h t$ (acheté).

- remplacement d'un son par un chiffre dont la dénomination porte la valeur phonétique recherchée: Koi de 9 ou Koi 29 (quoi de neuf).

- orthographe phonétique: dans l'exemple précédent, le remplacement de "qu-" par " $k$ " répond à la valeur phonétique, censée être univoque, de " $k$ " face à la graphie "c" et au digramme "qu-"; d'ailleurs, cela donnerait davantage un "look SMS" au message ${ }^{12}$. Il en est de même pour l'emploi de "z" à la place du "s" intervocalique: bizou (bisou), biz (bises), par hazar (par hasard). D'autres digrammes et trigrammes connaissent le même sort: "au", "eau" deviennent "o": ossi (aussi), bo (beau). Il s'agit là, d'une part, de supprimer des lettres "inutiles" et, d'autre part, d'établir une correspondance unique entre phonème et graphie ${ }^{13}$.

Plusieurs travaux (Anis 2001, Karsenti 2011, Georgakopoulou 2011) mettent en évidence l'hybridation entre l'écrit de l'oral des écrits numériques en général ${ }^{14}$. Pour nous, ce qui ressort des corpus est le principe phonographique évoqué qui donne à ces échanges l'apparence d'un oral scriptural ou d'un écrit oralisé et qui compense l'absence de la kinésique et du para-verbal de la communication orale en face-à-face.

12 Certains procédés traités comme des cas de simplification (Fairon et al. $2006: 36$ ) sont, en réalité, des transformations qui contribuent à donner ce même aspect SMS: qwa (quoi), parfwa (parfois), aujourdwi (aujourd'hui). Pour les auteurs, ce serait dû à la prononciation belge $[\mathrm{w}]$ du graphème w (et non [v]). Il est possible aussi que le digramme "wh" des mots anglais (where, what,...) ait pu jouer comme emprunt graphique simplifié aux diphtongues françaises "oi" [wa], "ui" [yi], à moins que les usagers connaissent le symbole phonétique de la semi-consonne, $[\mathrm{w}] \ldots$

13 Plus rarement, on a trouvé l'effet d"'écrasement" dû à des assimilations consonantiques: chui (je suis), ché pas (je ne sais pas) (Fairon, et al., 2006: 37). C'est une transposition à l'écrit de l'articulation réelle, phénomène qui relève également de l'orthographe phonétique, dans la mesure où l'on se sert d'un digramme existant pour rendre compte d'un segment sonore insécable, autrement dit, on utilise un digramme unique pour un phonème unique.

14 Tandis que Véronis \& Guimier de Neef (2006) n’y voient dans la communication médiée que de "nouvelles formes de communication écrite". Quant à Georgakopoulou A. (2011), elle est citée par Marcoccia (2016): "Computer-Mediated Communication", In J-O. Östman et J. Verschueren (eds.), Pragmatics in practice, Amsterdam, John Benjamins publishing: 93-110. 


\section{Bilan}

Ces mécanismes de phonétisation sont-ils nouveaux? Fairon et al. (2006: 50) nous rappellent que l'on trouve des traces de ce procédé à une époque où la téléphonie portable était peu ou pas connue, par exemple dans la manière d'écrire K7 pour cassette. Dans une œuvre majeure de la littérature française, Zazie dans le métro (1959), R. Queneau s'amuse à utiliser, parmi d'autres procédés linguistiques, une orthographe phonétique qui se veut le reflet de la langue parlée: Skeutadittaleur pour Ce que t'as dit tout à l'heure en est un exemple.

De fait, la question sur l'écriture, phonétique ou bien étymologique du français, ainsi que des autres langues européennes, s'est posée dès la fin du $X^{\mathrm{e}}$ siècle. Le latin, le grec et l'hébreu étaient des langues de culture, tandis que les langues vernaculaires étaient les langues de communication. En pleine Renaissance, les Umanisti tels qu'Érasme ${ }^{15}$ défendaient l'extension et l'expression de tous les savoirs en langue vulgaire, car la langue constitue pour l'homme le moyen privilégié de sa relation au monde et avec autrui. Le $\mathrm{XVI}^{\mathrm{e}}$ siècle est donc le siècle de l'élaboration des premières grammaires des langues d'Europe, nécessaires dès lors que ces langues, véhiculées par une littérature, allaient être enseignées dans les cours européennes. La standardisation des langues vernaculaires a été une initiative novatrice, dans laquelle l'orthographe et la prononciation ont pris une place capitale.

Depuis le passage à l'écrit des langues romanes, vers le $\mathrm{XI}^{\mathrm{e}}$ siècle, il a fallu recourir à des signes existants ou inventés pour rendre tous les sons qui n'avaient pas d'équivalent en latin. Ainsi, les clercs et les scribes auxquels on confiait la copie des actes et des manuscrits du Moyen Âge écrivaient leur texte avec une orthographe dépourvue de signes superflus: c'était une écriture phonétique, même si elle changeait souvent d'aspect par manque de grammaires et donc de normalisation.

Dans la fixation de l'orthographe du français au $\mathrm{XVI}^{\mathrm{e}}$ siècle, deux courants se sont opposés: les réformateurs, qui défendaient l'écriture phonétique (parmi lesquels on comptait des grammairiens, lexicographes ou écrivains tels que Maigret, Peletier du Mans, Ramus, Ronsard) et les partisans de l'orthographe étymologique (Abel Mathieu, Robert Estienne - nommé imprimeur royal par François $\mathrm{I}^{\mathrm{er}}$ ). C'est cette dernière qui l'a emporté et elle est caractérisée par l'ajout de lettres plus ou moins étymologiques qui ont parfois une fonction distinctive (ex.: mais vs. mes, lait vs. laid).

Plusieurs tentatives de réformes du français, plus ou moins fructueuses, se sont succédé depuis, allant toutes dans le même sens phonographique, en supprimant notamment des lettres étymologiques et muettes et en rapprochant ainsi l'orthographe de la prononciation. Parmi elles, citons celle du poète Jean Godard, en 1642, ou celle de l'abbé d'Olivet qui, en 1740, concerna environ 5000 mots qui ont figuré sous leur forme nouvelle dans la troisième édition du dictionnaire de l'Académie française. Plus près de nous, en 1990, rappelons que

$15 C f$. Érasme (1525). 
les recommandations de rectifications émanant du Conseil supérieur de la langue française ont concerné 1500 mots et ont ainsi conduit à une harmonisation des verbes en -eler et -eter (il ruissèle, j'époussète): il s'agit là d'un exemple des efforts de simplification de l'orthographe qui se sont soldés uniquement par la coexistence des normes et des usages ${ }^{16}$.

D'autres langues, telles que l'italien et l'espagnol, ont une écriture plus phonétique que le français. Or, à notre connaissance aucun alphabet des langues naturelles ne présente une correspondance univoque avec les phonèmes de la langue orale. C'est ce qui explique, d'ailleurs, la création de l'Alphabet Phonétique International (API), par l'Association des phonéticiens français et britanniques en 1888. Cet alphabet assigne un symbole unique à chaque segment sonore insécable, de façon à pouvoir transcrire les principales réalisations phonétiques des différentes langues du monde (ainsi, les multigrammes on, -ont, -ons correspondent au seul symbole: [õ]).

Les minimessages des jeunes ont pris parti pour une orthographe phonétique qui n'est pas totalement stable, non pas par manque de grammaires comme au Moyen Âge mais grâce à la liberté de création et à la dimension ludique que lui insufflent ses scripteurs. Les analyses de corpus et des dictionnaires de SMS montrent, en effet, une grande variété de formes graphiques pour le même vocable. Ainsi, le mot aujourd'hui peut être répertorié de plusieurs façons selon le mécanisme à l'œuvre:

- Squelette consonantique: ojd,

- Troncation: aujourd, auj,

- Phonétisation: ojourd8,

si bien qu'il est difficile d'établir une norme de la communication écrite médiée par portable.

\section{3. Économie du langage}

Dans les messages SMS on constate un autre mécanisme récurrent qui consiste à réduire les phrases et les mots. Pourtant, de nos jours, la plupart des forfaits téléphoniques offrent des SMS illimités; les smartphones sont, de surcroît, dotés d'un écran tactile avec clavier inséré pour faciliter l'encodage des messages. Or, les écrans restent de petite taille et les usagers ont toujours besoin de transmettre les messages rapidement!

Dans l'ensemble des corpus ont été relevés des siglaisons, des acronymes, des abréviations et des troncations. Parmi toutes ces formes du système typologique, les sigles ne foisonnent pas. Pourtant, S.M.S sont les sigles en anglais de "Short Message System" que 1'on peut traduire littéralement par "Système de Message Succinct". Cette définition du message court vient du fait qu'à l'origine, les messages S.M.S ne pouvaient pas dépasser un certain

16 Pour plus d'informations, voir "Les Rectifications de l'orthographe" (Journal officiel de la République française, $\mathrm{n}^{\circ}$ 100, 6 décembre 1990) et Mots (1991), $\mathrm{n}^{\circ}$ 28, Orthographe et société, Catach, N., Petitjean, L. et Maurice Tournier M. (dirs). 
nombre de caractères, 160 en l'occurrence. Les sigles les plus répandus sont: Rstp (réponds s'il te plaît), Raf (rien à faire), Ras (rien à signaler), $C t$ (c'était).

Les acronymes sont encore plus rares: askip (à ce qu'il paraît), $M D R$ (mort de rire). Quant à la célèbre formule des minitextos $L O L$, de l'anglais "Laughing out" (rire bruyamment), elle est née dans les années 1980, lorsqu'il y avait très peu de téléphones portables en circulation.

En revanche, les abréviations sont nombreuses: plus spécifiquement ce sont des raccourcissements d'un mot ou d'un groupe de mots en veillant à ce qu'il reste reconnaissable. On les appelle des "squelettes consonantiques" car la nouvelle unité graphique est faite des consonnes du mot ou du syntagme en éliminant les voyelles: Slt (salut), stp (s'il te plaît), bjr (bonjour), tjr (toujours), Dak (d'accord, d'acc), tkt (t'inquiète). Certaines étaient universellement admises bien avant la pratique linguistique des SMS et font partie du français normé: etc. (et cætera), Mme (Madame): elles ont été récupérées dans la messagerie instantanée pour leur nombre réduit de lettres.

Parmi les abréviations on trouve des troncations, la chute de segments pouvant être à l'initiale ou à la fin des mots: ordi (ordinateur), grasse mat (grasse matinée), tain (putain).

Des transcatégorisations, au niveau de la morphosyntaxe, peuvent avoir lieu si elles permettent d'abréger le mot: Sms-moi (envoie-moi un sms), il suffit de me smser le plus tot poss (il suffit de m'envoyer un SMS le plus tôt possible), Je pe dodo dans tes bras? (je peux dormir dans tes bras?), je trouve lol l'histoire avec caro (je trouve 'amusante à mourir de rire' l'histoire avec Caro).

On arrive même à l'omission de mots grammaticaux, tels que les déterminants: $D e$ main, j'étudie pr [les] examens et les syntagmes je n'ai et il y a: Pas eu mon exam... (je n'ai pas eu mon examen), J'entend rien pcq trop de bruit (je n'entends rien parce qu'il y a trop de bruit), qui font penser au style télégraphique.

Enfin, certains néographismes relèvent à la fois de la phonétisation des caractères et de l'abréviation, tels que la simplification des consonnes doubles $b b$ (bébé), et l'utilisation de $K$ à la place de c et qu: c moi ki t'fé cet efé la? (c'est moi qui te fais cet effet-là?).

Et, malgré l'apparition des emojis sur les nouveaux claviers digitaux, les émoticônes sont toujours employées :-), ;-), :D, :-* (sourire, clin d'œil, rire, bisou, respectivement).

\section{Bilan}

Siglaisons, acronymes, abréviations et troncations permettent d'accélérer l'encodage du message envoyé. Mais est-ce que ce sont des créations SMS? Sans parler des sigles et des acronymes, tellement répandus dans la langue française, on constate, d'une part, que sont attestées des formes utilisées, depuis longtemps, dans la prise rapide de notes ( $b c p$, $t$, $t j r, p d t)$; d'autre part, beaucoup de mots tronqués standard en langage familier, ou pas, sont 
même devenus usuels, notamment ceux qui sont constitués d'un faux préfixe emprunté à un substantif ou à un adjectif grec ou latin (métro, moto, vélo, ciné ont pratiquement remplacé chemin de fer métropolitain, motocyclette, vélocipède et cinématographe).

L'ensemble de ces mécanismes n'est donc pas nouveau dans l'histoire du langage. Le principe de l'économie du langage, décrit par le fonctionnaliste français André Martinet (1960), désigne l'un des principaux mécanismes d'évolution en linguistique. Il a été transposé dans le terrain de la phonétique comme la loi du moindre effort articulatoire. Dans le langage, comme dans n'importe quelle activité humaine, il existe une tendance naturelle à minimiser l'effort investi. La minimisation de l'effort qui, on l'a vu, se manifeste de ces différentes façons pour transmettre l'information, est observable depuis des temps immémoriaux.

Les Romains mêmes et les copistes médiévaux étaient très enclins à abréger pour économiser du papier et de l'encre, des produits onéreux. La devise SENATUS POPULUSQUE ROMANUS, signifiant "Le Sénat et le peuple romain", souvent abrégée sous la forme du sigle SPQR, était l'emblème de la République romaine puis, par tradition, de l'Empire romain.

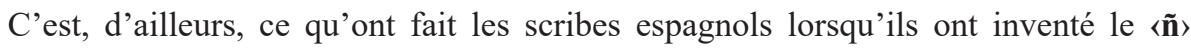
comme abréviation du digramme 〈nn〉 (lat. MONTAGNA > esp. médiéval montanna > esp. moderne montaña).

D'autres exemples dans l'histoire du langage montrent des réductions des mots: les signes alchimiques contenues dans l'Encyclopédie de Diderot, classés par Ponot (1971), sont formés de lettres et de chiffres: N ('Ana'); 5 ('Quinte Essence') et d'alliances de symboles et de lettres, difficiles à transcrire ici tels qu'ils apparaissent dans les manuscrits grecs, où ils ont originairement été rencontrés. Comme chacun sait, les symboles de la chimie contemporaine, ont progressivement remplacé l'alchimie: $H 2 O$ (2 atomes d'hydrogène et un atome d'oxygène ou “eau"), CO2 (un atome de carbone et deux atomes d'oxygène).

Ponot étudie les signes alchimiques comme éléments constitutifs d'un langage. Il explique (1971: 66) que l'alchimie était une doctrine hermétique, que "les alchimistes étaient [...] des hommes de passion acharnés à arracher ses secrets à la nature" mais "leur art était maudit et [...] il devait être voilé de mystère", pour éviter que le peuple l'utilise vulgairement et lui enlève toute sa valeur.

À propos de cette notion d'hermétisme, de secret et de mystère, il y a lieu de se demander si l'eSMS présente cette même propriété pour les non-initiés. Pour certains adultes la décodification de ces messages peut s'avérer aussi difficile que les argots, mais à des degrés divers selon la maîtrise et la connaissance qu'a chacun des pratiques évoquées.

\section{Expressivité et créativité}

Les typologies scripturales établies par les auteurs cités précédemment révèlent des usages qui ont une fonction expressive au sens qu'a défini Jakobson (1963), dans la mesure 
où le message, centré sur le locuteur, exprime essentiellement les sentiments et ainsi qu'elle est définie dans le dictionnaire: "Expressif: Qui exprime bien, qui traduit d'une manière suggestive une façon d'être, un sentiment, une pensée" (TLF).

D'une part, dans des contextes à contenu affectif, on y trouve fréquemment des onomatopées ou des interjections comme pfff, grrr, ooooh, hmmmmm. Mais aussi des mots étirés, pratique facilitée probablement par les forfaits sans limite de caractères des nouveaux mobiles: ke jeu taimmmmmeu, a plusssssssss, gros bisouxxx/bizzzoux, JT ADOOOR, kissss a 2m1, C moiiii, Et EFFFACE MON NUMERo, revien VITTTE et des répétitions des signes de ponctuation: Als t ou??????????????, koi ????????????, TU PEUX ME TEL!!!!!!!!!!!

C'est dans ce même type de contexte, dans lequel prime l'expression des affects, que les usagers construisent des liaisons en " $\mathrm{n}$ " et en "s" évoluant en agglutinations: lez zamours ca va? (les amours, ça va?), Bisoux mn nange ke j'aime bcp bcp cré fort, Ztadorrre trop fort. Ces procédés scripturaux ayant un côté d"'emphase graphique", imitent, en même temps, le langage oral enfantin, en remplaçant, par exemple, “j” par “z", "très" par "cré". En effet, les enfants en phase d'acquisition du langage ont le réflexe de remplacer les sons difficiles à prononcer par des sons plus faciles, ici [3] par [z], [tr] par [kь]. De plus, le style "communication enfantine" est communément associé à la déclaration des émotions spontanées et sincères.

Enfin, la généralisation d'un pluriel en "-oux" pour les termes affectueux: bisoux, kissoux, calinoux, poutoux, chouchoux soulignent non seulement le côté ludique et créatif de la communication par SMS, mais aussi la tendance à l'analogie linguistique qui est propre également aux plus jeunes locuteurs. D'ailleurs, certaines graphies liées à des réminiscences de bandes dessinées vont dans le même sens: po au lieu de l'adverbe "pas" étant une habitude langagière du héros de BD Titeuf: G tj po u la lettr, tu la envoyé a kel adres? (j'ai toujours pas eu la lettre, tu l'as envoyée à quelle adresse?).

D'autre part, des traces de verlan et de code-switching ne sont pas exclues des corpus de SMS: sa me fait déprimer a donf (ça me fait déprimer à fond); digramme "oo" pour "ou": bizoo et kikoo (bisou, coucou). Cougnon (2011), Morel\& Pekarek Doehler (2013) et Morel (2017) se sont aussi intéressés à l'eSMS comme un lieu privilégié pour le déploiement de pratiques plurilingues. Dans le corpus de ce dernier, ayant le français comme langue de base, $14,15 \%$ des occurrences comportent au moins un cas de code-switching. Tous arrivent à la même conclusion : l'alternance codique est une ressource qui augmente l'expressivité des messages. Des emprunts à l'anglais mais aussi à l'espagnol, à l'italien, à l'allemand et au suisse allemand sont utilisés “dans les ouvertures (Hi! Hallo! Hola!) et les clôtures de messages (bye, bis dann, see you), mais aussi pour des voux (good luck), des termes d'adresses (buddy, boy, bella, miss, señor, amore) et d'affection (bacio, ti amo)". Ces termes, bien spécifiques des interactions par SMS, côtoient des emprunts plus routinisés employés à l'oral dans les échanges informels: Ouf,je me faisais du souci,redonne-moi des news demain,bisoux. 


\section{Bilan}

En bref, les éléments propres aux interactions orales, telles que l'intonation, les exclamations, le langage corporel, sont suppléés dans les écrits SMS non seulement par les émojis et les émoticônes, mais aussi par une série de procédés scripturaux tels que: onomatopées, interjections, allongement des formes, majuscules, agglutinations, nouvelles suffixations. Ce type de communication se dote ainsi d'une fonction expressive et affiche de bonnes doses de créativité, ce qui est propre au langage humain. Dans le déploiement du code-switching, cette expressivité va de pair avec l'affichage, par les scripteurs, d'une identité cosmopolite et translinguistique.

\section{Dimension sociolinguistique}

Pour les sociolinguistes variationnistes, les langues peuvent être soumises à des variables liées au contexte social (Labov, 1972) et, de façon générale, à des paramètres sur lesquelles les individus fondent leur identité, tels que l'âge, le sexe, l'ethnie, la religion, la profession, le groupe.

La langue véhiculée dans les SMS est une variation diaphasique (ou situationnelle) et diastratique à la fois. En effet, parmi les styles ou les registres de langue que les jeunes maîtrisent, ils peuvent mettre à contribution - par écrit, via leur smartphone - l'eSMS dans la situation de communication "requise" (variation diaphasique). Or, cette situation a toujours lieu entre pairs, c'est-à-dire, que l'usage de la langue que font ces locuteurs est déterminé par le groupe social auquel ils se sentent appartenir: la communication par SMS peut, à ce titre, être considérée comme un sociolecte (variation diastratique).

Or, l'écriture SMS est devenue un rituel de socialisation de la plupart d'utilisateurs de smartphone. Entre adultes en général et dans le monde professionnel, elle reste conventionnelle, en particulier depuis l'arrivée des téléphones intelligents. En revanche, les jeunes et les adultes en contexte familier cultivent clairement une orthographe qui leur est propre et ce, en dépit des logiciels d'écriture intuitive - conçus pour réduire le temps consacré à la rédaction d'un message - et des forfaits mobiles avec SMS illimités qu'offrent la plupart des opérateurs de télécommunications.

Il est indéniable que les scripteurs ont, plus ou moins consciemment, un désir de transgression du registre normé ${ }^{17}$ de la langue mais motivé par quoi? D'un côté, c'est un gain de temps et d'espace car l'eSMS permet d'échanger davantage et de transmettre plus d'information de façon très condensée. D'un autre côté, il n'est en rien comparable à la fonction

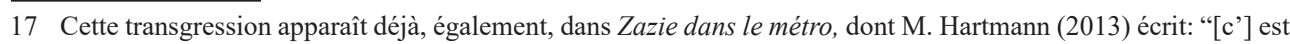
un roman insolent à l'égard de la belle langue française. Il introduit un autre rapport au langage, irrespectueux des commandements de la grammaire, de la syntaxe et de l'orthographe. Le roman combine néo-français et français classique". 
subversive de sociolectes tels que le verlan, principal élément du français contemporain des cités: face à l'intentionnalité sociale qui définit ce dernier (Bachman \& Basier, 1984: 184), la communication électronique a quelque chose de fortuit, provoqué par l'ère du numérique et la banalisation des technologies de l'information et de la communication qui s'en est suivie.

Est-ce que la transgression de la norme linguistique symbolise-t-elle une appartenance sociale ou culturelle? Au vu de la place que ces conversations instantanées ont pris dans la vie de tous les jours, nous y voyons plutôt un style de communication par lequel sont concernés tous ceux qui, appartenant à un monde numérique et globalisé, se trouvent en contexte familier; ce style de socialisation dépasse les frontières des locuteurs d'une langue donnée $e^{18}$.

\section{Conclusions}

D’un point de vue sociolinguistique, un nouveau mode de communication est né. Lié à la révolution numérique du début du $\mathrm{XXI}^{\mathrm{e}}$ siècle, il est uniquement pratiqué à l'écrit tout en tendant à refléter les usages de l'oral. Il s'est adapté à toutes les conversations écrites médiées par téléphone mobile. Ce type d'interaction, développé par notamment par les jeunes, est caractérisé par des variations orthographiques par rapport à la langue normée mais combinées avec des formes standard et illustre, une fois de plus, que le changement est une propriété inhérente aux langues.

Ce phénomène graphique met en exergue deux principes qui régissent le langage humain: la phonétisation de l'écriture et l'économie du langage, qui se traduisent par l'éclosion de néographismes pas tout à fait surprenants. En abrégeant les formes, les utilisateurs mettent à l'honneur la loi du moindre effort scriptural.

Aussi, les usagers de SMS ont réagi à l'abondance de lettres quiescentes ou à l'absence du principe phonographique. Nos alphabets apparaissent déroutants dans la mesure où ils enseignent et compilent les graphies, pas les sons d'une langue. Or une langue est orale avant tout, notion dont on a perdu la conscience dans nos sociétés alphabétisées, d'où l'incohérence des alphabets car en nommant les lettres on leur attribue un seul son, alors qu'un son peut être transcrit avec des combinaisons différentes de lettres! L'observation du langage des textos pointe du doigt la nécessité d'une réflexion pédagogique au niveau de l'école et des lexicographes (n'en déplaise aux virtuoses de l'orthographe) qui mette davantage l'accent sur la différence entre deux matériaux sémiotiques: le phonique et le graphique.

Le code SMS remplit une fonction expressive qui lui permet, presque, de se substituer

18 Il faudrait, bien sûr, des analyses comparatives des discours numériques de différentes langues, comme celles réalisées par M. Bieswanger (2007), cité par Marcoccia (2016): “2 abbrevi8 or not 2 abbrevi8: A Contrastive Analysis of Different Space and Time-Saving Strategies in English and German Text Messages" in T. Hallett, S. Floyd, S. Oshima \& A. Shield (eds.), Symposium About Language and Society-Austin (SALSA) XIV: 2006. Texas Linguistic Forum, Vol. 50. 
aux interactions orales grâce à un ensemble de procédés disorthographiques, y compris le code-switching. Or, concernant sa dimension cryptée, cette variété linguistique est d'une compréhension relativement accessible aux non-usagers, compte tenu de deux mécanismes ancestraux et récurrents qui en sont à la base: abréviations et phonétisation des caractères.

En termes d'identité sociale ou culturelle, cette étude porte sur des textos en français mais le phénomène de l'eSMS est d'une ampleur mondiale et susceptible de se développer dans toutes les communautés linguistiques séduites par l'industrie d'internet et de la téléphonie mobile et donc, dans une bonne quantité de langues (à titre d'exemple, WhatsApp est disponible dans plus de 40 langues et jusqu'à 60 langues sur le système d'exploitation Android $\left.{ }^{19}\right)$.

Les travaux notamment de Morel \& Pekarek Doehler (2013) déduisent de l'usage du plurilinguisme dans les échanges électroniques que "les scripteurs affichent leur appartenance à une communauté qui se veut translinguistique et globalisée". Ce qui est certain c'est que ces pratiques scripturales sont communes à un groupe d'individus beaucoup plus large que celui des argots des métiers ou des argots sociologiques, car elles concernent toutes les populations qui ont connu la révolution digitale et la banalisation des technologies de l'information et de la communication, à condition que cela se fasse entre pairs. Le choix de ce langage découle moins d'un désir de transgression de la norme que de l'avènement de l'ère numérique et des circonstances technologiques et matérielles qui ont poussé, dans un premier temps, à abréger l'écriture; ceci s'est avéré une tâche facile lorsque la plupart des langues ont des lettres superflues. Puis le besoin de communiquer rapidement a rejoint les aspects ludique et expressif de ce nouveau style de langage. Un tout pour conquérir, de façon ininterrompue, jeunes et moins jeunes.

\section{Références bibliographiques}

André, Frédéric. 2017. Pratiques scripturales et écriture SMS. Analyse linguistique d'un corpus de langue française, thèse de Doctorat, Université Paris-Sorbonne.

Anis, Jacques. 2001. Parlez-vous texto? Guide des nouveaux langages du réseau. Paris, Le Cherche Midi.

Anis, J., DE Fornel, M., Fraenkel, B. (2004, organisateurs). "La communication électronique: Approches linguistiques et anthropologiques" in Colloque international, EHESS, Paris, 5-6 février 2004.

Auzanneau, M. \& Juillard C. 2012. "Jeunes et parlers jeunes: catégories et catégorisations" in Langage et Société n 141, 5-20.

Bachmann, Ch. et Basier L. 1984. "Le verlan: argot d'école ou langue des Keums?". In Mots, $\mathrm{n}^{\circ}$ 8. Numéro spécial. L'Autre, l'Etranger, présence et exclusion dans le discours. 69-187, DOI : https://doi.org/10.3406/mots.1984.1145.

19 Information tirée du site de WhatsApp: <https://faq.whatsapp.com/fr/general/426771995837866/>. 
Banegas SaOrín, Mercedes. 2012. "La description des sons dans les premières grammaires pour l'enseignement du français diffusées en Espagne au XVI ${ }^{\mathrm{e}}$ siècle: Meurier et Sotomayor" in Les langues étrangères: apprentissages et pratiques en Europe (1450-1700), Zuili, M. et Baddeley S. (dirs), Paris, Presses de l’Université Paris-Sorbonne (PUPS), 229-243.

Bernicot, J., Goumi, A., Bert-Erboul, A., Volckaert-Legrier, O. 2014. "How do skilled and less-skilled spellers write text messages? A longitudinal study of sixth and seventh graders Running title: Text messages in teenagers" in Journal of Computer Assisted Learning, Wiley, 2014, 30 (6), 10.1111/jcal.12064. hal-01392433.

Boyer, Henri. 2001. "Le français des jeunes vécu/ vu par les étudiants. Enquêtes à Montpellier, Paris, Lille" in Langage et société 2001/1 n 95, 75-87. https://www.cairn.info/revuelangage-et-societe-2001-1-page-75.htm.

Clarin (journal argentin) : https://www.clarin.com/ediciones-anteriores/cv-sms_0_Sy7NA80RaKg.html [25/08/2019].

Collas, Aurélie. “Écrire 'SMS' ne nuit pas à l'orthographe” in Le Monde, 19/03/2014, https://www.lemonde.fr/education/article/2014/03/19/ecrire-sms-ne-nuit-pas-a-1-orthographe_5995062_1473685.html [10/06/2019].

Combes, Céline. 2014. Étude du coût cognitif de l'écriture SMS chez les adolescents. Psychologie. Université Toulouse le Mirail - Toulouse II, 2014. Français. NNT: 2014TOU20014. tel-01134181.

Cougnon, Louise-Amélie. 2010. "Orthographe et langue dans les SMS. Conclusions à partir de quatre corpus francophones" in Ela. Etudes de linguistique appliquée, 160 (4), 397-410. https://www.cairn.info/revue-ela-2010-4-page-397.htm.

Cougnon, L.-A. \& François, T. 2011. "Étudier l>écrit SMS. Un objectif du projet sms4sciences” in A. Stähli \& al. (Eds.), La communication par SMS en Suisse. Usages et variétés linguistiques. Themenheft, Linguistik Online.

DARMANIN, Jules. 2015. “oklm”, “msk”, “jpp”: petit lexique du nouveau langage SMS des ados, Le Figaro, 19/06/2015 : http://www.lefigaro.fr/secteur/high-tech/2015/06/19/3200120150619ARTFIG00150-oklm-msk-jpp-petit-lexique-du-nouveau-langage-sms-des-ados. php?redirect_premium\&_scoop_post $=$ ec821511-3b62-11e5-e029-00221934899c\& scoop_topic $=5032239 \#$ _ scoop_post $=$ ec821511-3b62-11e5-e 029-00221934899c\& scoop_topic=5032239 [consulté le 20/12/2019]

Ducrot, O. \& Todorov, T. 1972. Dictionnaire encyclopédique des sciences du langage. Paris, Seuil

Duretz, Marlène. 2018. "L'Instagram des plus beaux textos d'amour et des déclarations enflammées" in Le Monde, 09/02/2018 <https://www.lemonde.fr/m-perso/article/2018/02/09/1-instagram-des-declarations-enflammees_5254388_4497916.html> [01/04/2020].

D\&V Translation Agency <https://www.dvtranslation.com/blog/apprendre-le-langage-smsen-trois-etapes/> [10/04/2020]

Érasme. 2002 [1525]. La langue, traduction de Jean-Paul Gillet. Genève, Labor et Fides.

Fairon, C., Klein, J.-R., PAumier S. 2006. Le langage SMS. Étude d'un corpus informa- 
tisé à partir de l'enquête "Faites don de vos sms à la science”. UCL, Louvain-la-Neuve, Presses Universitaires.

Fairon, C., \& Klein, J.-R. 2010. "Les écritures et graphies inventives des SMS face aux graphies normées" in Le Français aujourd'hui, 170(3),113-122.

GoudAILler, Jean-Pierre. 2007. "Français contemporain des cités: langue en miroir, langue du refus" in Adolescence, 2007/1, n 59, 119-124, DOI: 10.3917/ado.059.0119. <https://www. cairn.info/revue-adolescence1-2007-1-page-119.htm>.

Hartmann Marie. 2013. “Zazie dans le métro. Impertinences et désenchantements"in Roman 20-50, 2013/2, n 56, 157-167. DOI: 10.3917/r2050.056.0157.<https://www.cairn.info/ revue-roman2050-2013-2-page-157.htm>.

JAKOBSOn, Roman. 1963. Essais de linguistique générale, T. 1. Paris, Éditions de Minuit.

JND, Le Journal du Net: <https://www.journaldunet.com/management/ressources-humaines/1069468-postuler-a-une-offre-d-emploi-par-sms/> [16/07/2019].

Karsenti, T. \& Collin, S. 2011. "Le mal d'écrire: la faute aux textos?" in Québec français, $\mathrm{n}^{\mathrm{o}} 163,82-83$.

Kogkitsidou Eleni. 2018. Communiquer par SMS: Analyse automatique du langage et extraction de l'information véhiculée, thèse de Doctorat en Linguistique. Université Grenoble Alpes. <http://tel.archives-ouvertes.fr/tel-01968698>.

Labov, William. 1976. Sociolinguistique. Paris, Minuit.

La revue des médias, INA: <https://larevuedesmedias.ina.fr/revolution-numerique-les-journalistes-face-au-nouveau-tempo-de-linfo $>$ [05/04/2020].

LIÉNARD, Fabien. 2008. “Analyse linguistique et sociopragmatique de l'écriture électronique. Le cas du SMS tchaté” in Gerbault, J. (éd.) La langue du cyberespace: de la diversité aux normes. Paris, L'Harmattan, 265-274.

Marcoccia, Michel. 2016. Analyser la communication numérique écrite. Paris, Armand Colin, "I.COM", DOI: 10.3917/arco.marco.2016.01. <https://www.cairn.info/analyser-la-communication-numerique-ecrite--9782200602987.htm>.

Martinet, André. 1960. Éléments de linguistique générale. Paris, Armand Colin.

Morel, Étienne \& Pekarek Doehler Simona. 2013. “Les 'textos' plurilingues: l'alternance codique comme ressource d'affiliation à une communauté globalisée" in Revue française de linguistique appliquée, 2013/2, vol. XVIII, 29-43, DOI: 10.3917/rfla.182.0029. $<$ https://www.cairn.info/revue-francaise-de-linguistique-appliquee-2013-2-page-29.htm>.

Morel Étienne. 2017. Textos: assemblages hététosémiotiques. Louvain-la-Neuve, De Boeck, Neveu, Franck. 2004. Dictionnaire des sciences du langage. Paris, Armand Colin.

PAnCKhURST Rachel. 2009. "Short Message Service (SMS): typologie et problématiques futures" in Polyphonies, pour Michelle Lanvin, Université Paul-Valéry Montpellier 3, 33-52, hal-00443014. 
Panckhurst, R., \& Moïse, C. 2012. "French text messages. From SMS data collection to preliminary analysis" in Linguisticae Investigationes, 35(2), 289-317.

Panckhurst, R., Detrie, C., Lopez, C., Moïse, C., Roche, M. et Verine, B. 2014. 88 milSMS. A corpus of authentic text messages in french. Produit par l'Université Paul-Valéry Montpellier III et le CNRS, en collaboration avec l'Université catholique de Louvain, financé grâce au soutien de la MSH-M et du Ministère de la Culture (Délégation générale à la langue française et aux langues de France) et avec la participation de Praxiling, Lirmm, Lidilem, Tetis, Viseo. ISLRN: 24-713-187-947-8.

Ponot, René. 1971. "Les signes alchimiques" in Communication \& Langages, $\mathrm{n}^{\circ}$ 12, 65-79, $<$ https://www.persee.fr/doc/colan_0336-1500_1971_num_12_1_3903>.

VÉronis, J. \& GUIMIER De NeEF, E. 2006. "Le traitement des nouvelles formes de communication écrite" in Sabah, G. (éd.), Compréhension automatique des langues en interaction. Paris, Hermès Science, 227-248 [05/04/2020].

\section{Annexe I}

CORPUS extrait de Cédrick FAIRON, Jean RENÉ et Sébastien PAUMIER (2006), p. 31, sq.

\begin{tabular}{lll}
\hline Phénomène & Exemple SMS & Équivalent français normé (parlé) \\
\hline Phonétisation & Je t'm & Je t'aime \\
des carac- & J'espère ke CT sympa & J'espère que c'était sympa \\
tères: lettres et & g pac la pir nui d ma vi & J'ai passé la pire nuit de ma vie \\
chiffres & Un petit cl1 d'œil & Un petit clin d'œil \\
& A12C4 & À un de ces quatre! \\
& Kl1 & Câlin \\
& 7 swaré & Cette soirée \\
& 2day & Today \\
& 2night & Tonigth \\
& JV encor devoir 10QT & Je vais encore devoir discuter \\
\hline Orthographe & Je l'ai pa vue & Je l'ai pas vue \\
& par hazar & par hasard \\
& G acheté ls cado de noel & J'ai acheté les cadeaux de Noël \\
& Ta beaucoup de dvoir? & T'as beaucoup de devoirs? \\
& j'voulé allé ché toi & Je voulais aller chez-toi \\
& c moi ki t'fé cet efé la? & C'est moi qui te fais cet effet-là? \\
& Kand tu veux & Quand tu veux \\
& Pourkoi tu téléfones pa? & Pourquoi tu téléphones pas? \\
& Je te fais des bizou & Je te fais des bisous \\
\hline
\end{tabular}




\begin{tabular}{|c|c|c|}
\hline & PootooXx & Poutous \\
\hline & mon namooreu & mon amoureux \\
\hline & Tu m mank mon ti loo & Tu me manques, mon petit loup \\
\hline & Dm1 tu fai qwa bb? & Demain tu fais quoi, bébé? \\
\hline & Bone swaré & Bonne soirée \\
\hline & J swi a la mutuel & Je suis à la mutuelle \\
\hline & G parfwa peur & J'ai parfois peur \\
\hline & $\mathrm{g}$ bswdètr prè dtoi & j'ai besoin d'être près de toi \\
\hline & Tu veu passer aujourdwi ??? & Tu veux passer aujourd'hui??? \\
\hline & ce soir chui en forme & ce soir je suis en forme \\
\hline & ché pas si on tla di & je ne sais pas si on te l'a dit \\
\hline & Ztadorrre trop fort & Je t'adore trop fort \\
\hline & ztèm mon Nounours & je t'aime, mon nounours \\
\hline \multirow{23}{*}{$\begin{array}{l}\text { Phénomènes } \\
\text { graphiques } \\
\text { onomatopées } \\
\text { interjections, } \\
\text { mots étirés, } \\
\text { répétitions de } \\
\text { signes de ponc- } \\
\text { tuation, } \\
\text { liaisons évoluant } \\
\text { en agglutinations }\end{array}$} & \multicolumn{2}{|l|}{ pfff } \\
\hline & \multicolumn{2}{|l|}{ grrr, } \\
\hline & \multicolumn{2}{|l|}{ ooooh, } \\
\hline & \multicolumn{2}{|l|}{ hmmmmm } \\
\hline & \multicolumn{2}{|l|}{ ke jeu taimmmmmeu } \\
\hline & a plussssssssss & à plus! \\
\hline & \multicolumn{2}{|l|}{ gros bisouxxx / bizzzoux } \\
\hline & JT ADOOOR & Je t'adore \\
\hline & \multicolumn{2}{|r|}{ Kis, à demain } \\
\hline & \multicolumn{2}{|r|}{ C'est moi } \\
\hline & Et EFFFACE MON NUMERO Et efface mon numéro & $\begin{array}{l}\text { Et efface mon numéro } \\
\text { reviens vite }\end{array}$ \\
\hline & \multicolumn{2}{|l|}{ Als t ou??????????????? } \\
\hline & \multicolumn{2}{|l|}{ koi????????????? } \\
\hline & \multicolumn{2}{|l|}{ TU PEUX ME TEL!!!!!!!!!!! } \\
\hline & \multicolumn{2}{|c|}{$\begin{array}{l}\text { Bisoux mn nange ke j'aime Bisous, mon ange que j'aime beaucoup } \\
\text { bcp bcp cré fort }\end{array}$} \\
\hline & \multicolumn{2}{|c|}{ Kikou mn namoureu chéri $\quad$ Coucou, mon amoureux chéri } \\
\hline & lez zamours ca va? & les amours, ça va \\
\hline & \multirow{2}{*}{\multicolumn{2}{|c|}{$\begin{array}{l}\text { Jte raconte pa les zieux d pois- Je te raconte pas les yeux de poisson } \\
\text { son } \mathrm{k} \text { jme tape } \\
\text { que je me tape }\end{array}$}} \\
\hline & & \\
\hline & \multicolumn{2}{|r|}{ Salut! Ça va? } \\
\hline & \multicolumn{2}{|r|}{ Bonjour } \\
\hline & \multicolumn{2}{|r|}{ Tu peux répondre, s'il te plaît? } \\
\hline & Dak & D’accord \\
\hline
\end{tabular}




\begin{tabular}{lll}
\hline Rébus, icônes et & mobi* & Mobistar \\
symboles divers & tu te x malin & Tu te crois malin \\
& $:-) ;-$ :D :-* & Sourire, clin d'œil, bisou \\
& $+=$ & Plus, égal \\
& Tu ora 63,10€ (promis -> du) & Tu auras 63,10€ (ce qui est promis est \\
& phone pa -> maman assé éner- dû) \\
& vé & Ne téléphone pas car maman est assez \\
& & énervée \\
\hline Phénomènes & Tain j’tourne mal & Putain, je tourne mal \\
lexicaux: & pti prob d ordi et d internet & petit problème d'ordinateur et d'inter- \\
abrègement, & on a fait la grasse mat & net \\
sigles & RAS & on fait la grasse matinée \\
& & Rien à signaler \\
\hline Morphosyntaxe & Sms-moi qud tu arriv / il suffit & Envoie-moi un SMS quand tu arrives / \\
& de me smser le plus tot poss & il suffit de m'envoyer un SMS le plus \\
& & tôt possible \\
& Je pe dodo dans tes bras? & Je peux dormir dans tes bras? \\
& Demain, j’étudie pr examens... & Demain, j'étudie pour les examens... \\
& Pas eu mon exam... & Je n'ai pas eu mon examen... \\
& J'entend rien pcq trop de bruit. & Je n'entends rien parce qu'il y a trop \\
& oki pr semaine proch, ms ca- de bruit \\
formes & mion est déjà parti & Ok pour la semaine prochaine, mais le \\
& Livre archit déposé boite. Mer- camion est déjà parti \\
& ci pr pret. PhL & Livre d'architecture déposé dans ta \\
& h.e.t / Ht & boîte. Merci pour le prêt. Philippe \\
\hline & & aujourd'hui \\
& poutous \\
& & acheter \\
\hline & & \\
& & \\
& &
\end{tabular}




\section{Annexe II}

\section{Extrait du CORPUS CeRCA}

(Bernicot, Goumi, Bert-Erboul et Volckaert-Legrier (2014)

1 Apel moi si tu recoi ce message emeline (Appelle-moi si tu reçois ce message, Emeline)

2 Ya rien de drol (Y a rien de drôle)

3 Jen ai mar mn pere juste psk lordi lenerve c moi quil engueule (J'en ai marre mon père juste parce que l'ordinateur l'énerve c'est moi qu'il engueule)

4 Cc je voulai prendre de t nouvel (Coucou je voulais prendre de tes nouvelles)

5 Je c pa psk ma mere es de nui mn père du matin et du cou c mon oncle et ma tata qui von dormir he moi come sa je serai pa toute seule cet nui donc je c pa (Je sais pas parce que ma mère est de nuit mon père du matin et du coup c'est mon oncle et ma tata qui vont dormir chez moi comme ça je serai pas toute seule cette nuit donc je sais pas)

6 Je croier que tu devez mapeler (Je croyais que tu devais m'appeler)

7 Je sui conecter (Je suis connectée)

8 Ba aten jai pa menger (Bah attends j'ai pas mangé)

9 Cc tufe koi (Coucou tu fais quoi)

10 Je croi que jai la gripe (jai de la fièvre et je tousse bocoup donc jai plus de voie (Je crois que j'ai la grippe (j'ai de la fièvre et je tousse beaucoup donc j'ai plus de voix)) 
